\title{
Comparação de cinesioterapia em solo e em água em idosos
}

\author{
Comparison of cinesiotherapy and hydrotherapy in the elderly
}

\author{
Aline Cristina Tavares ${ }^{1}$, Tatiana Sacchelli ${ }^{2}$
}

\begin{abstract}
RESUMO
Introdução. O envelhecimento está associado com perda da capacidade funcional. Como conseqüência, há diminuição da independência e aumento de risco de quedas. Objetivo. Avaliar a melhora do desempenho funcional no idoso, comparando dois protocolos: em solo (S) e em água (A). Método. Um estudo prospectivo de 12 semanas, randomizado e cego foi realizado no departamento de fisioterapia da Universidade Metodista de São Paulo (UMESP). Os dados foram obtidos pela versão brasileira do questionário OARS (Older Americans Resources and Services Program), Brazilian Multidimensional Functional Assessment Questionnaire (BOMFAQ). Resultados. A pontuação média do OARS demonstrou passou de $70,6 \pm 1$ para $72,1 \pm 1(p=0,37)$ no grupo $S$ e de $71,5 \pm 0,8$ para $72,9 \pm 0,8(p=0,16)$ no grupo $A$. Uma atividade em $S$ (andar no plano) e uma atividade em $A$ (deitar e levantar da cama) apresentaram melhora significativa de $4,7 \pm 1,2$ para $5,6 \pm 0,7$ com $p=0,03$ de $4,7 \pm 1,2$ para $5,6 \pm 0,7$ com $p=0,02$, respectivamente. Conclusão. Um programa de atividade física baseada em exercícios em solo e em água aumenta significantemente a capacidade de andar no plano e de deitar e levantar da cama.
\end{abstract}

Unitermos. Envelhecimento, Força Muscular, Equilíbrio Musculosquelético.

Citação. Tavares AC, Sacchelli T. Comparação de cinesioterapia em solo e em água em idosos.

Trabalho realizado na Universidade Metodista de São Paulo. Clínica Escola-Modelo. São Bernardo do Campo - SP, Brasil.

1.Pós-Graduanda em Fisioterapia Hospitalar na Universidade Metodista de São Paulo - UMESP, São Bernardo do Campo - SP, Brasil.

2.Fisioterapeuta, Mestre em Ciência do Movimento, Professora do Curso de Graduação em Fisioterapia da UMESP, São Bernardo do Campo - SP, Brasil.

\section{SUMMARY}

Introduction. Aging is associated with decreased physical functional performance. As a consequence, there is loss of independency and risk of falls increase. Objective. To evaluate the improvement of functional performance in healthy elders, by comparing two protocols: on the ground (S) and in the water (A). Method. A 12-week, randomized, single-blind study was provided in the department of physiotherapy of the São Paulo Methodist University (UMESP). The data were gathered with a Brazilian version of OARS (Older Americans Resources and Services Program), Brazilian Multidimensional Functional Assessment Questionnaire (BOMFAQ). Results. The OARS score went from $70.6 \pm 1$ to $72.1 \pm 1(p=0.37)$ in group $S$ and from $71.5 \pm 0.8$ to $72.9 \pm 0.8(p=0.16)$ in group $A$. One activity on $S$ (walking on the plane) and one activity on A (lying and getting out of bed) have shown significant improvement from $4.7 \pm 1.2$ to $5.6 \pm 0.7(p=0.03)$, and from $4.7 \pm 1.2$ to $5.6 \pm 0.7$ $(p=0.02)$ respectively. Conclusion. A program based on exercises on the ground and in the water improves the capacity of walking on the plane and of lying and getting out of bed.

Keywords. Aging, Muscle Strength, Musculoskeletal Equilibrium.

Citation. Tavares AC, Sacchelli T. Comparison of cinesiotherapy and hydrotherapy in the elderly.
Endereço para correspondência: Aline Cristina Tavares Universidade Metodista de São Paulo R. Londrina, 658 CEP 09181-160, Santo André-SP, Brasil. E-mail: alinet84@gmail.com

Recebido em: 15/01/2009 Revisado em: 16/01/2009 a 05/08/2009

Aceito em: 06/08/2009

Conflito de interesses: não 


\section{INTRODUÇÃO}

Mais de $85 \%$ da população é constituída de idosos, que podem ser classificados como geronte, (indivíduo saudável), ou geriopata, (indivíduo doente). Em ambos, o envelhecimento conduz a uma perda progressiva das aptidões funcionais do organismo ${ }^{1}$ como a diminuição da capacidade aeróbica máxima e da tolerância ao exercício físico ${ }^{2}$; da potência ${ }^{3}$ e força muscu$\operatorname{lar}^{1,3,4}$, a redução da velocidade de reflexos, da coordenação ${ }^{2}$ e do equilíbrio e a sarcopenia ${ }^{5}$.

Entres essas alterações funcionais, as que desencadeiam maior dependência durante o desempenho da atividade funcional são o declínio da força muscular e do equilíbrio ${ }^{4-6}$. Todas as tarefas requerem um limiar da força para serem desempenhadas e o controle postural proporciona melhor estabilidade durante o desenvolvimento delas $s^{4,7}$.

Esse declínio funcional ${ }^{2,5}$ leva à maior incidência de morbidades com quedas ${ }^{8}$ e deterioração psicológica do idoso e aumento dos custos com encargos sociais para combatê-lo6.

Diversos estudos buscam a melhora das AVDs (atividade de vida diária) com exercícios que envolvem mobilizações articulares, transferências ${ }^{6}$, exercícios resistivos, treino de equilíbrio ${ }^{9}$, treino de marcha, além de recomendações para mudança do ambiente doméstico ${ }^{8}$ e próprio desenvolvimento de AVD's $^{9,10}$ em solo ${ }^{6-10}$. Entretanto, a capacidade de desempenhar exercícios em água, impossíveis ou difíceis de serem realizados em solo, resulta em efeitos psicológicos favoráveis que elevam a confiança e a moral do idoso ${ }^{11}$.

O objetivo deste estudo é avaliar a melhora da atividade funcional em idosos submetidos à cinesioterapia em solo e compará-lo com as respostas em água.

\section{MÉTODO}

\section{Amostra}

Este é um estudo prospectivo realizado entre os anos 2004 e 2005 na Clínica de Fisioterapia da Universidade Metodista de São Paulo (UMESP), após liberação do Comitê de Ética e Pesquisa (numero do protocolo).

Foram incluídos trinta e sete indivíduos acima de 64 anos de idade, sedentários, que compreendessem e assinassem o termo para participar do estudo. Foram excluídos indivíduos com doenças neurológicas ou cadeirantes. Assim, os participantes selecionados ao trabaIho foram locados, de forma randomizada (com sorteio de envelope lacrado para o grupo pertencente), em grupo para intervenção no solo (total de dezessete participantes) o e outro para intervenção em água (total de vinte participantes).

\section{Avaliação}

Antes da intervenção e ao final de 24 sessões (2 semanais, com duração de 60 minutos cada) de tratamento os pacientes eram avaliados por um profissional não envolvido diretamente com o estudo, a fim de evitar resultados tendenciosos com o uso da versão brasileira do OARS (Older Americans Resources and Services Program): Brazilian Multidimensional Functional Assessment Questionnaire (BOMFAQ) ${ }^{12}$, conforme suas habilidade em deitar e levantar da cama comer pentear o cabelo (C), andar no plano (D), tomar banho (E), vestir-se (F), ir ao banheiro em tempo sem que haja perda urinária ou fecal (G), subir um lance de escadas $(\mathrm{H})$, medicar-se na hora $(\mathrm{I})$, andar perto de casa $(\mathrm{J})$, fazer compras $(\mathrm{K})$, preparar refeições $(L)$, cortar as unhas dos pés $(M)$, sair da condução (N), e fazer limpeza de casa (O),

A pontuação de cada pergunta do questionário foi dada de acordo com o auto-relato de cada participante, sendo 0 (zero) quando ele referia não realizar uma determinada atividade resultante de uma dificuldade muito intensa ou não saber realiza-la, 2 (dois) quando essa dificuldade era muita, 4 (quatro) quando pouca e 6 (seis) quando ausente.

O protocolo da intervenção em solo foi realizado através de aquecimento, alongamento, treino de equilíbrio e alongamento. $\mathrm{O}$ aquecimento consistia em andar de frente com passos amplos e com movimentos alternados de flexão de ombro a $90^{\circ}$ ( 1 minuto); andar de frente com flexão de $90^{\circ}$ de quadril e de joelho alternando movimentos de membros superiores (1 minuto); andar de lado com passos amplos associado à movimentação lateral de membros superiores (1 minuto para cada lado); jogar bola com o terapeuta (1 minuto). No alongamento eram realizados exercícios com extensores de joelho ( 1 de cada lado), extensores de quadril (1 minuto de cada lado) e extensores de tronco (1 minuto de cada lado). Durante o fortalecimento 
( 1 minuto e 15segundos de cada lado) eram utilizados extensores de quadril, extensores de tronco superior, extensores de joelho e extensores de tronco inferior. Ao longo do Treino de equilíbrio (2 minutos e 30 segundos cada exercício nos quais as instabilizações eram realizadas pelo quadril) o indivíduo permanecia sentado na bola sem aproximar os pés da bola e ou apoiar as mãos sobre a bola ou coxas, de modo que não se fixasse; exercício com a prancha de equilíbrio; deambulação sobre o espaguete; exercício com oscilação anterior realizada pelo fisioterapeuta; exercício com oscilação posterior realizada pelo fisioterapeuta, com apoio de antebraço na região anterior e posterior do tronco do paciente; exercício com mini-tramping em ortostatismo com apoio unilateral; exercício em pé com apoio em retropé, exercício com minitramping jogando bola com o fisioterapeuta; exercício no balancim. Por fim, durante o relaxamento (1 min cada exercício) foi realizada tração de cervical, tração lombar, fricção, massagem com bola.

O protocolo da intervenção em água também foi realizado através de aquecimento, alongamento, fortalecimento e relaxamento. Durante o Aquecimento eram realizados exercícios de andar de frente (1 minuto) e depois de costas (1 minuto) com passos amplos e com movimento anteroposterior dos membros superiores na superfície da água com 2 halteres pequenos, andar de lado com passos amplos e com movimento lateral de membros superiores (MMSS) (1 minuto para cada lado), andar de frente com flexão de $90^{\circ}$ de quadril, joelho e tornozelo alternando movimento de MMSS, em dissociação de cinturas (1 minuto). Na fase de Alongamento ( 2 minutos de cada exercício) alongavam-se os extensores de quadril, joelho e tronco. O Fortalecimento se focava nos músculos extensores de joelho, tronco superior, tronco inferior e quadril (2minutos e 30segundos cada exercício). Durante o Treino de equilíbrio (2minutos e 30segundos cada exercício nos quais as instabilizações eram realizadas por turbulência do fisioterapeuta ora na região anterior, ora na posterior ou ainda nas laterais, devendo retornar à linha média) sentado na bola com tríplice flexão de membros inferiores (MMII) em pé sobre um dos membros e joelhos semi-flexionados; sentado de frente sobre o apoio do fisioterapeuta na região de escápulas ou ísquios sem apoio dos pés ao chão com oscilações para laterais; sentado de costas sobre o apoio do fisioterapeuta na região palmar sem apoio dos pés ao chão com oscilações para laterais; em pé sobre pranchas realizando flexoextensão de quadril; em pé sobre pranchas realizando abdução-adução; em pé sobre a prancha de propriocepção; em pé com oscilação posterior pelo tronco do paciente mantendo o peso somente nos calcanhares; Por fim, os exercícios de Relaxamento ( 1 minuto cada exercício com paciente em supino com flutuadores na cintura pélvica e em calcanhares) eram realizados com o paciente recebendo apoio na região occipital e inclinações da cervical como serpente; movimento de antepulsão e retropulsão de tronco como onda; tração de cervical durante a expiração do paciente; recebe turbulência em toda a região de tronco posterior.

\section{Análise Estatística}

As variáveis numéricas foram expressas por média, desvio padrão (DP) e mediana. A associação destas variáveis com os fatores de interesse foi verificada pelo teste $t$ de Student para amostras com distribuição normal. Para a comparação dos resultados antes e depois do tratamento foi utilizado o teste não paramétrico Kruskal Wallis. Níveis descritivos ( $p$ ) inferiores a 0,05 foram considerados estatisticamente significantes. Todos os testes utilizados foram executados pelo programa estatístico STATISTICA.

\section{RESULTADOS}

Não houve diferença entre os grupos $(p=0,1)$ quantoàidade,com média de $72 \pm 5$ anos deidadeno grupo que realizou intervenção em solo e de 70 $\pm 5,9$ no que recebeu a intervenção em água (Tabela 1).

Tabela 1: Dados demográficos de ambos os grupos $(p>0,05)$.

\begin{tabular}{|c|c|c|c|c|c|c|}
\hline \multirow{2}{*}{$\begin{array}{c}\text { Voluntá- } \\
\text { rios }\end{array}$} & \multicolumn{2}{|c|}{ Total } & \multicolumn{2}{c|}{ Grupo Solo } & \multicolumn{2}{c|}{ Grupo Água } \\
\cline { 2 - 7 } & $\mathrm{n}$ & Idade & $\mathrm{n}$ & Idade & $\mathrm{n}$ & Idade \\
\hline Homens & 7 & $73 \pm 3,4$ & 2 & $75,5 \pm 4,9$ & 5 & $73 \pm 3,4$ \\
\hline Mulheres & 30 & $71 \pm 6$ & 15 & $72 \pm 4,6$ & 15 & $69 \pm 6,3$ \\
\hline Total & 37 & $71 \pm 5,4$ & 17 & $72 \pm 5$ & 20 & $70 \pm 5,9$ \\
\hline
\end{tabular}


O grupo solo, após a intervenção, apresentou aumento da pontuação para a atividade Andar no Plano (de $4,7 \pm 1,2$ para $5,6 \pm 0,7$ com $p=0,03)$. As outras atividades não mostraram diferenças significantes (Tabela 2).

Ao analisar os dados correspondentes à atividade em água pode-se constatar que houve melhora da atividade deitar e levantar da cama (de 4,8 \pm 1 para $5,3 \pm 1$ ) sendo esta melhora estatisticamente significante ( $p=0,02$; Gráfico 1). Algumas das atividades apresentaram melhora após o programa, porém sem significância estatística. Foram elas: comer, pentear o cabelo, vestir-se, subir um lance de escada, preparar refeições fazer limpeza de casa.

Tabela 2. Comparação das médias de cada atividade pré e pós-intervenção fisioterapêutica nos dois grupos (solo e água).

\begin{tabular}{|l|l|l|l|l|}
\hline & \multicolumn{3}{|l|}{ Grupo Solo } & \multicolumn{2}{l|}{ Grupo Água } \\
\hline & Pré & Pós & Pré & Pós \\
\hline A-Cama & 4,8 & 5,1 & $4,8^{*}$ & $5,3^{*}$ \\
\hline B-Comer & 6 & 5,6 & 5,7 & 5,9 \\
\hline C-Pentear Cabelo & 5,9 & 5,5 & 5,6 & 5,8 \\
\hline D-Andar no plano & $4,7^{*}$ & $5,6^{*}$ & 5,1 & 5,1 \\
\hline E-Tomar Banho & 4,9 & 4,9 & 5,2 & 5,2 \\
\hline F-Vestir-se & 5,2 & 4,9 & 4,9 & 5,2 \\
\hline G-Banheiro em tempo & 5,4 & 5,4 & 5 & 5 \\
\hline H-Subir escada (1lance) & 4,2 & 4,6 & 4 & 4,1 \\
\hline I-Medicar-se na hora & 5,5 & 5,5 & 4,9 & 4,9 \\
\hline J-Andar perto de casa & 4,9 & 5,3 & 5,3 & 5,3 \\
\hline K-Fazer Compras & 4,2 & 4,2 & 5 & 4,5 \\
\hline L-Preparar refeições & 5,4 & 5,1 & 5,4 & 5,6 \\
\hline M-Cortar unhas dos pés & 2,8 & 2,1 & 2,7 & 2,7 \\
\hline N-Sair de condução & 4,3 & 4,8 & 4,7 & 4,6 \\
\hline O-Limpar casa & 2,4 & 2,9 & 3,2 & 3,7 \\
\hline *p=0,03 e \#p=0,02. & & & & \\
& & & & \\
\hline
\end{tabular}

As atividades que não se modificaram após a intervenção fisioterapêutica em solo (Gráfico 2) foram tomar banho, ir ao banheiro em tempo sem que haja perda urinária ou fecal, medicar-se na hora; e as que se mantiveram em água foram as descritas como andar no plano, tomar banho, ir ao banheiro em tempo sem que haja perda urinária ou fecal, medicar-se na hora, andar perto de casa e cortar as unhas do pé.

Dentre as atividades que apresentaram diminuição de sua pontuação, no entanto, diferença estatisticamente significan- te, cinco eram da avaliação em solo: comer, pentear o cabelo, vestir-se, preparar refeições, cortar as unhas dos pés, e duas das atividades apresentaram declínio após a intervenção em água: sair da condução e fazer compras (Gráfico 3).

\section{DISCUSSÃO}

O sexo feminino foi prevalente em ambos os grupos, provavelmente pelo fato de as mulheres viverem, em média, oito a mais que os homens ${ }^{13}$ ou pelo motivo delas demonstrarem maior preocupação com a saúde e, consequentemente, buscarem maior atendimento na área da saúde ${ }^{14}$. Já a não-desistência pode ter ocorrido devido a benefícios sociais e emocionais que são propostos pelo exercício ${ }^{10}$.

A ênfase do protocolo proposto se assemeIha com os de outros estudos, direcionados a idosos, já publicados ao fazer uso de aquecimento ${ }^{15}$, alongamento e resistência de grupos musculares antigravitacionais, e de equilíbrio ${ }^{8}$ uma vez que estão intimamente relacionados à funcionalida$\mathrm{de}^{10,15}$. A intervenção mais parecida e com o mesmo foco de objetivo descreve uma intervenção de 2 vezes semanais com 60 minutos cada, com duração total de 10 semanas, mas nessa experiência de 2001, há melhora significativa no desempenho funcional (porém não indicam o valor de $p$ achado para a avaliação do desempenho funcional) ${ }^{16}$. Eles, contudo, adicionaram ao tratamento uma sessão semanal de atividades laborais e sociais e seus idosos eram mais jovens que os do estudo presente (média de $63,6 \pm 6,3$ ). Esses dois fatores podem ter favorecido a melhora no desempenho funcional dos 20 idosos estudados.

Outros resultados também parecem conflitantes com os nossos, pois relata melhora significativa de força muscular e desempenho funcional pelo teste ADAP (Assessment of daily activity performance) mesmo com o número de participantes semelhantes ao nosso (24), com média de idade semelhante $74,6 \pm 4,8$ anos, e com protocolo de 10 minutos de aquecimento, 40 de exercício resistivo e dos demais 10 para desaquecimento ${ }^{10}$. Eles, no entanto realizavam exercícios resistivos e em seu protocolo e acrescentaram o treino de musculatura de membros superiores.

Contradizem-se também com o resultado de 
Gráfico 1. Comparação das atividades deitar e levantar d Gráfico 1. Comparação das atividades deitar e levantar da cama, comer, pentear o cabelo, andar no plano e tomar banho pré e pós avaliação segundo a pontuação do questionário versão brasileira do OARS (Older Americans Resources and Services Program).

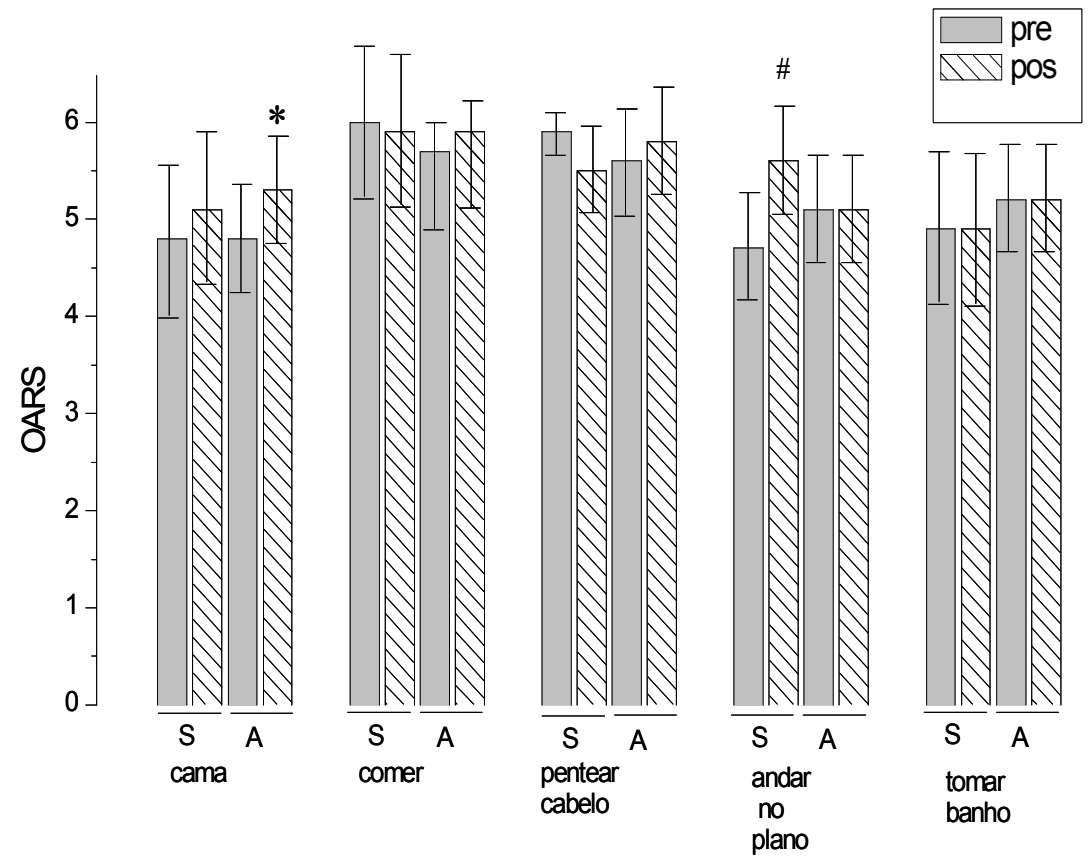

$S=$ grupo que recebeu intervenção em solo; $A=$ grupo que recebeu intervenção em água; * $p=0,02$ grupo $A$ e \# $p=0,03$ grupo $S$.

Gráfico 2. Comparação das atividades vestir-se, ir ao banheiro em tempo sem que haja perda urinária ou fecal, subir um lance de escadas, medicar-se na hora e andar perto de casa, pré e pós avaliação segundo a pontuação do questionário versão brasileira do OARS (Older Americans Resources and Services Program).

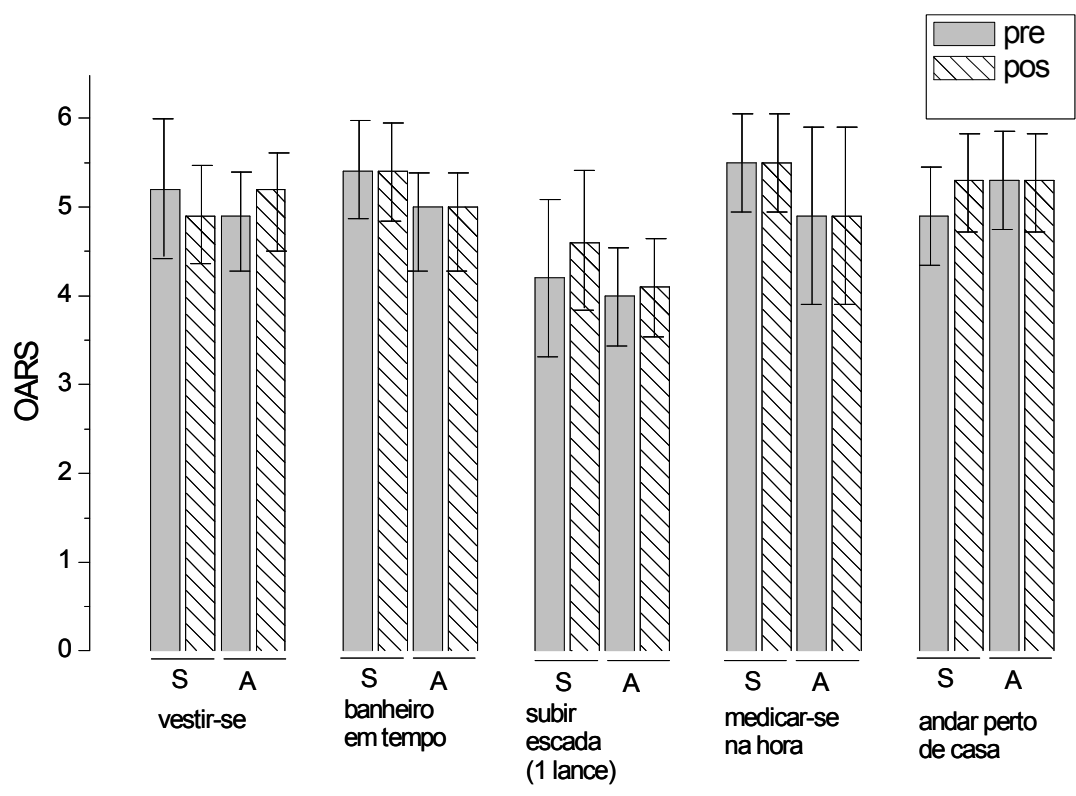

$S=$ grupo que recebeu intervenção em solo; $A=$ grupo que recebeu intervenção em água. 
Gráfico 3. Comparação das atividades fazer compras, preparar refeições, cortar as unhas dos pés, sair da condução e fazer limpeza de casa pré e pós avaliação segundo a pontuação do questionário versão brasileira do OARS (Older Americans Resources and Services Program).

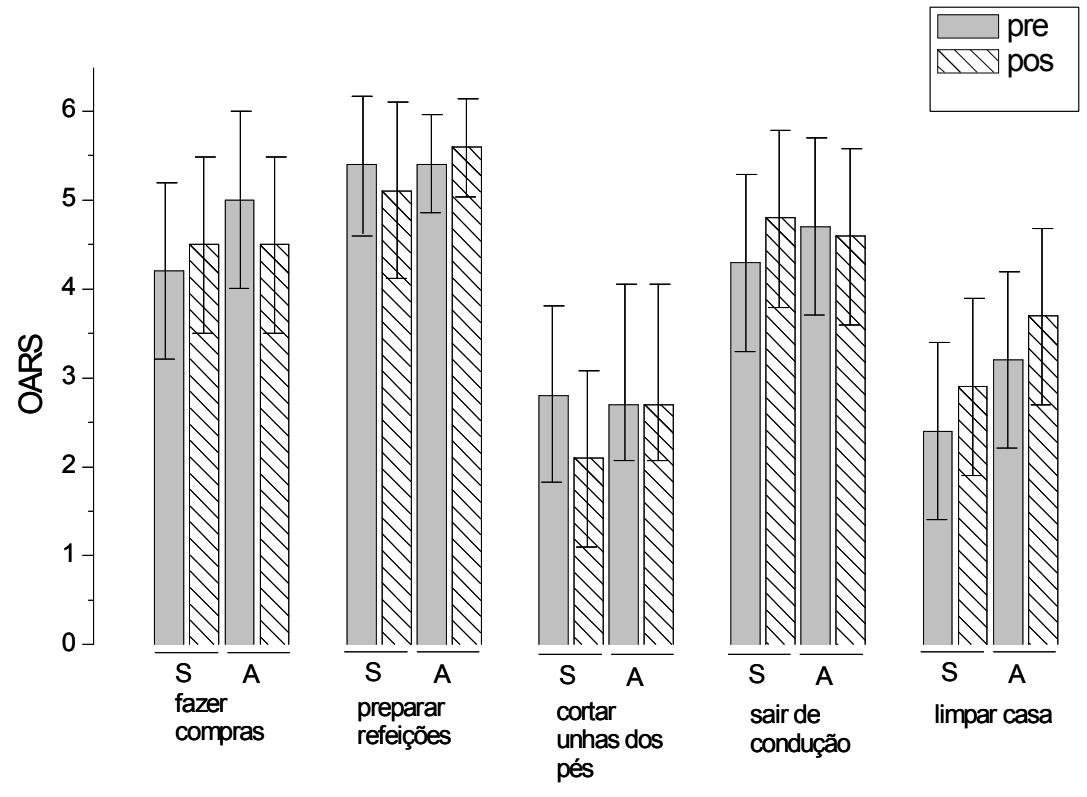

$\mathrm{S}=$ grupo que recebeu intervenção em solo; $\mathrm{A}=$ grupo que recebeu intervenção em água.

outro estudo, que mostra melhora funcional significativa, provavelmente porque o tempo de sua intervenção foi maior que o nosso, sete meses, e sua população apresentava nítido comprometimento de membros inferiores, uma vez que a inclusão ao estudo era de idosos incapazes de levantar-se da cadeira com os braços cruzados e que tivessem pontuação menor que 10s no teste de caminhada rápida, não específica para funcionalidade ${ }^{6}$. Melhoras significantes ao se comparar grupos em solo e em água no que se diz respeito à meIhora de equilíbrio já foram relatadas ${ }^{17,18}$. Somente um estudo apresentou achados semelhantes aos nossos, referindo que não houve diferença estatisticamente significante entre ambos os grupos de tratamento em água e solo após a intervenção ${ }^{17}$ As variáveis em que os pacientes apresentaram pior pontuação em comum, em ambos os grupos, foram as descritas como: a habilidade em deitar e levantar da cama, subir um lance de escadas, cortar as unhas dos pés, sair da condução, e fazer limpeza de casa. Isso pode ser explicado pelo fato de que a maioria das tarefas parece exigir melhor controle postural, e as conseqüências mais graves de um equilíbrio pobre, ocorrem na realização das AVD, já que a habilidade em manter o equilíbrio postural é essencial para manter uma performance eficiente em todo o tipo de atividade ${ }^{19-21}$.

Isso parece estar sustentado pelo resultado que as tarefas que não exigem muito do controle postural, mas sim da musculatura dos MMSS, descritas como comer, pentear o cabelo, ir ao banheiro em tempo sem que haja perda urinária ou fecal e preparar refeições apresentaram, nos dois grupos, as pontuações mais altas. . 
Levando em conta que o protocolo não direcionou qualquer exercício especificamente aos membros superiores, o ponto-chave para o desfecho parece ser o equilíbrio e força de MMII, assim como descrito em 2003 que a perda de massa e força muscular, decorrentes do processo natural de envelhecimento, ocorre com maior intensidade nos $\mathrm{MMII}^{22}$.

Durante as 24 sessões de treinamento, o processo de envelhecimento não foi interrompido, assim é provável que os participantes teriam declínio funcional relacionado aos fatores intrínsecos do envelhecimento e decorrentes da inatividade ${ }^{1,9,10,15}$. Dessa forma, a própria manutenção do desempenho representa um alto benefício para este grupo de idosos.

\section{REFERÊNCIAS}

1.Timo-laria C, Irigoyen C, Krieger EM. Fisiologia do envelhecimento. In: Petroianu A, Pimenta LG. Clínica e cirurgia geriátrica. Rio de Janeiro:Guanabara Koogan. 1999, p54-8.

2.Checorrch M, Smith E. Effects of aging on bone. In: Kauffman TL. Geriatric Rehabilitation Manual. Edinburgh: Churchill Livingstone., 1999, p12-4.

3.Skelton DA, Beyer N. Exercise and injury prevention in older people. Scand J Med Sci Sports. 2003;13:77-85.

4.Marks R, Allegrante J, MacKenzie CR, Lane J. Hip fracture among the elderly: cases, consequences and control. Ageing 2003;2:57-93.

5.Frontera WR, Larsson L. Skeletal muscle function in older people. In: Kauffman TL. Geriatric Rehabilitation Manual. Edinburgh: Churchill Livingstone, 1999, p8-11.

6.Gill Y, Baker D, Gottschlk M, Peduzzi P, Allore H, Nes P. A prehabilitation program for the prevention of functional decline: effect on highter-level physical function. Arch Phys Rehabil. 2004;85:1043-9.

7.Seguin R, Nelson, ME. Os benefícios do treinamento de força em adultos. Am J Preven Med 2003;25:141-9.

8.Brawley LR, Rejeski J, King A. Promover atividade física para idosos. Os desafios para mudanças comportamentais. Am J Prevent Med 2003;25:172-83.

9.Frank JS, Aftab E, Patla AE. Balance and mobility challenges in older adults. Implications for preserving community mobility. Am J Prevent Med 2003;25:157-63.

10.Vreede P, Samson M, Meeteren N, Bom JV, Duursma S, Verhaar $\mathrm{H}$. Functional tasks exercise versus resistance exercise to improve daily function in older women: a feasibility study. Arch Phys Med Rehabil 2004;85:1952-61.

11.Campion MR. Os efeitos fisiológicos, terapêuticos e psicólogos da atividade aquática. In: Campion MR. Hidroterapia. Princípios e práticas. São Paulo: Manole, 2000, p174.

\section{CONCLUSÃO}

Conclui-se que um programa de atividade física baseada em exercícios em solo e em água aumenta significantemente a capacidade de andar no plano e de deitar e levantar da cama. É possível que os baixos resultados encontrados nesse estudo sejam conseqüência de uma amostra pequena, e as melhoras poderiam ser significantes caso os exercícios recebessem maior resistência ou se o tempo de intervenção fosse maior. Novos trabalhos devem ser realizados com maior número de paciente e em comparação com grupo controle ou outros protocolos, a fim de obter maiores informações sobre o tema

12.Blay LS, Ramos RL, Mari J. Validity of a Brazilian Version of the Older Americans Resources and Services (OARS) Mental Health Screening Questionnaire. JAGS 1988;36:687-92.

13.Camargos MCS, Perpétuo IHO, Machado CJ. Expectativa de vida com incapacidade funcional em idosos em São Paulo. Rer Panam Salud Pub 2005;17:5-6.

14.Michel JP, Robine JM. A "new" general theory of population ageing. Genev Pap Risk Insur 2004;29:667-75.

15.Santos LD, Samela LFT, Lelis FO, Lobo MB. Eficácia da atividade física na manutenção do desempenho funcional do idoso. Rev Bibliogr Fisioter Bras 2001;2:169-77.

16.Salmela L, Santos L, Goulart F, Cassiano J, Hirochi T. Efeitos de atividades físicas e terapêuticas em adultos maduros e idosos. Fisioter Bras 2001;12:99-106.

17.Douris P, Southard V, Varga C, Schauss W, Gennaro C, Reiss A. The effect of land and aquatic exercise on balance scores in older adults. J Geriatr Phys Ther 2006;26:3-6.

18.Simmons V, Hansen PD. Effectiveness of water exercises on postural mobility in the well elderly: an experimental study on balance enhancement. J Gerontol 1996;51:M223-7.

19.Daubney ME, Culham EG. Lower-Extremity Muscle Force and Balance Performance in Adults Aged 65 Years and Older. Phys Ther 1999;79:1177-85.

20.Era P, Avlund K, Jokela J, Gause-Nilsson I, Heikkinem E, Steen $B$, et al. Postural Balance and Self-Reported Functional Ability in 75-Year-

21. Horak FB, Henry SM, Shumway-Cook A. Postural Perturbations: New Insights for Treatment of Balance Disorders. Phys Ther 1997;77:517-33.

22.Shumway-Cook A, Woolacott MH. Envelhecimento e Controle Postural. In: Shumway-Cook A, Woollacott MH. Controle Motor. Teoria e Aplicações. São Paulo: Manole, 2003, p209-31. 